\title{
SIMULAÇÃO DOS EFEITOS DAS MUDANÇAS DO USO DA TERRA NA DINÂMICA DE CARBONO NO SOLO NA BACIA DO RIO PIRACICABA ${ }^{1}$
}

\author{
ANDRÉA MARIA SILVEIRA ${ }^{2}$, REYNALDO LUIZ VICTORIA ${ }^{3}$, MARIA VICTORIA BALLESTER ${ }^{4}$, \\ PLIINIO BARBOSA DE CAMARGO ${ }^{5}$, ${\text { LUIZ ANTONIO MARTINELLI }{ }^{5} \text { e MARISA DE CÁSSIA PICCOLO }}^{6}$
}

\begin{abstract}
RESUMO - Neste trabalho teve-se como objetivo testar o modelo CENTURY para simular os efeitos das mudanças de uso da terra nos teores de carbono no solo e na produção primária líquida na bacia do rio Piracicaba, SP. O modelo foi parametrizado e as simulações foram realizadas considerando-se áreas de florestas, pastagens e cana-de-açúcar, dada a sua importância econômica. Os resultados obtidos indicaram que a alteração do uso/cobertura do solo provoca a diminuição do estoque de carbono do solo. As simulações realizadas na transição de uma floresta para cana-de-açúcar indicaram um decréscimo de $28 \%$ no estoque de carbono nos primeiros 12 anos, e diminuição de $42 \%$ com 50 anos de cultivo do solo com essa gramínea. Na simulação da transição de uma floresta para pasto, seguida do cultivo da cana-deaçúcar, verificou-se que na primeira mudança (floresta-pastagem) a perda de carbono foi de $24 \%$, enquanto na segunda alteração (pastagem-cana) a perda foi de $22 \%$. Com relação à produção primária, os resultados obtidos de floresta $\left(6,6 \mathrm{t} \mathrm{ha}^{-1}\right.$ ano $\left.^{-1}\right)$, cana-de-açúcar $\left(77,82,80 \mathrm{t} \mathrm{ha}^{-1}\right.$ ano $\left.^{-1}\right)$ e pasto $\left(6,5 \mathrm{t} \mathrm{ha}^{-1}\right.$ ano $\left.^{-1}\right)$, mostraram-se similares aos valores observados no campo por outros autores.
\end{abstract}

Termos para indexação: biogeoquímica, modelagem, subtropical.

\section{SIMULATION OF THE EFFECTS OF LAND USE CHANGES IN SOIL CARBON DYNAMICS IN THE PIRACICABA RIVER BASIN, SÃO PAULO STATE, BRAZIL}

\begin{abstract}
The objective of this study was to test adequability of the CENTURY model to simulate the effects of land use changes on soil carbon content and net primary production to specific subtropical Brazilian environmental conditions. After parameterization, simulations were performed for forested areas, pasture and sugarcane cultures which have economical importance and representative areal extension in the Piracicaba River basin, São Paulo State, Brazil. The results obtained indicate that changes in use/cover from forest to agricultural practices resulted in lower soil carbon stocks. The simulations of the transition from forest to sugarcane indicated a decrease of $28 \%$ in soil carbon stocks for the first 12 years and $42 \%$ for 50 years of sugarcane cultivation. The simulation of the transition from forest to pasture and then to sugarcane resulted in a $24 \%$ loss of soil carbon stock for the first transition (forest-pasture) and of $22 \%$ for the second one (pasture-sugar-cane). The net primary production results obtained for forest $\left(6.6\right.$ ton $\left.\mathrm{ha}^{-1} \mathrm{yr}^{-1}\right)$, sugarcane $\left(77,82,80\right.$ ton $\left.\mathrm{ha}^{-1} \mathrm{yr}^{-1}\right)$ and pasture ( 6.5 ton $\mathrm{ha}^{-1} \mathrm{yr}^{-1}$ ) were similar to those observed by other authors in field measurements.
\end{abstract}

Index terms: biogeochemistry, modelling, subtropical.

${ }^{1}$ Aceito para publicação em 8 de junho de 1999.

${ }^{2}$ Analista de Sistemas, M.Sc., CENA-USP, Caixa Postal 96, CEP 13400-970 Piracicaba, SP. E-mail: andrea@carpa.ciagri.usp.br

${ }^{3}$ Eng. Agrôn., Ph.D., Prof. Titular, CENA-USP.

${ }^{4}$ Bióloga, Dr., CENA-USP.

${ }^{5}$ Eng. Agrôn., Dr., CENA-USP.

${ }^{6}$ Quím. Ind., Dr., CENA-USP.

\section{INTRODUÇÃO}

A transformação de áreas de vegetação natural em áreas de cultivo implica mudanças na estrutura e no funcionamento dos ecossistemas. Por exemplo, modificações no uso da terra podem acelerar a erosão e as perdas de solo por escoamento, alterar o 
número e o tipo de organismos, e interferir na dinâmica da matéria orgânica do solo (Cerri, 1986; Trumbore et al., 1995).

O estabelecimento de políticas de desenvolvimento agrícola e industrial no Brasil, em sua grande maioria, é determinado por fatores econômicos, sociais e políticos. As alterações funcionais e estruturais nos ecossistemas, que poderão surgir em decorrência de tais atividades, são, muitas vezes, ignoradas ou relegadas a um segundo plano. Um dos principais aspectos que contribui para este tipo de comportamento é a dificuldade em prever alterações em escalas locais, regionais e globais, em um tempo compatível com a agilidade dos investimentos econômicos. A simulação de sistemas pode preencher essa lacuna, tornando-se um instrumento importante nesse tipo de previsão (Steyaert, 1993; Wheeler, 1993; McNulty et al., 1997).

Atualmente, existem diferentes modelos de simulação de ecossistemas: CENTURY (Parton et al., 1987), Terrestrial Ecosystem Model (TEM) (Melillo et al., 1993), Carnegie Ames Stanford Approach (CASA) (Potter et al., 1993), Carbon Assimilation in the Biosphere Model (CARAIB) (Warnant et al., 1994), Land Surface Model (LSM) (Bonan, 1995) e outros.

O modelo CENTURY obteve sucesso no seu uso em diversos locais, na interpretação dos dados de campo para previsões a curto e longo prazo, onde foi determinado o impacto de diferentes práticas de manejo da matéria orgânica do solo (Carter et al., 1993; Parton \& Rasmussen, 1994). Parton et al. (1988) utilizaram o CENTURY para simular o impacto do cultivo de trigo na dinâmica da matéria orgânica, mineralização de nutrientes e produção da planta durante 100 anos. O modelo representou corretamente as mudanças nos níveis de C, N, P e S orgânico e inorgânico causados pelo cultivo de trigo. Em outro trabalho, Schimel et al. (1996) aplicaram o modelo CENTURY para uma série de florestas e pradarias distribuídas globalmente, para examinar a variabilidade do $\mathrm{C}$ do solo em grande escala. Este modelo foi desenvolvido para as condições de clima temperado e tem sido pouco testado em condições subtropicais.

Em regiões tropicais, a conversão da floresta em áreas agrícolas ou com vegetação secundária vem sendo apontada como um dos processos fundamen- tais que afetam a dinâmica do $\mathrm{C}$, as fontes e sumidouros dos gases do efeito estufa $\left(\mathrm{CO}_{2}, \mathrm{CH}_{4}\right.$ e $\left.\mathrm{N}_{2} \mathrm{O}\right)$, os fatores controladores do clima e dos processos ecológicos. Para elucidar o papel de tais mudanças, é necessário estabelecer como elas afetam os padrões de produção e mineralização da matéria orgânica e a ciclagem de nutrientes. Este trabalho teve como objetivo testar o modelo CENTURY para simular os efeitos das mudanças de uso da terra nos teores de carbono do solo e na produção primária, na bacia do rio Piracicaba, em São Paulo.

\section{MATERIAL E MÉTODOS}

\section{Área de estudo}

A parametrização do modelo CENTURY e as simulações foram testadas em área natural de floresta subtropical e em áreas cultivadas com pastagens e cana-de-açúcar, que têm importância econômica e significativa área plantada no Estado de São Paulo, especialmente, na bacia do rio Piracicaba (São Paulo, 1994).

A bacia do rio Piracicaba tem uma extensão de $12.400 \mathrm{~km}^{2}$, com aproximadamente $90 \%$ de sua área no Estado de São Paulo e 10\% no Estado de Minas Gerais, apresentando um crescimento econômico elevado e acelerado (São Paulo, 1994).

O clima da região é subtropical, com temperaturas médias anuais entre $18^{\circ} \mathrm{Ce} 22^{\circ} \mathrm{C}$. Somente a região situada na serra da Mantiqueira possui clima temperado, com temperatura média anual entre $12^{\circ} \mathrm{C}$ e $18^{\circ} \mathrm{C}$ (São Paulo, 1990). A distribuição pluvial segue um regime típico da região subtropical com verão chuvoso, que se estende de outubro a março, e inverno seco, de abril a setembro. A média pluviométrica anual da bacia é de $1.440 \mathrm{~mm}$; e desse total, aproximadamente $70 \%$ retornam para a atmosfera, via evapotranspiração, e o restante é escoado pelos rios (Pellegrino, 1995).

As principais atividades em termos econômicos na bacia do Piracicaba são a pastagem, a qual ocupa, atualmente, $50 \%$ da sua área, e a cultura da cana-de-açúcar, que representa $24 \%$ dessa área. As áreas de florestas estão reduzidas a pequenos fragmentos, restando apenas $6 \%$ da cobertura original.

\section{Modelo CENTURY 4.0}

Esse modelo é o produto de esforços de pesquisas que inicialmente pretendiam desenvolver um modelo geral da dinâmica do sistema solo-planta para as pradarias da 
América do Norte (Parton et al., 1987, 1988). Atualmente, o CENTURY está sendo utilizado em simulações de produção primária, ciclagem de nutrientes e dinâmica da matéria orgânica do solo para sistemas de pradarias, áreas agrícolas e florestas em várias regiões do mundo (Parton et al., 1987; Sanford Junior et al., 1991).

Os principais submodelos e equações que compõem o modelo CENTURY e a maneira como os coeficientes dessas equações foram determinados encontram-se descritos em Parton et al. (1987), e são descritos resumidamente a seguir:

\section{Submodelo de matéria orgânica do solo}

Considera que a matéria orgânica do solo (MOS) na camada de 0-20 cm de profundidade é composta por três diferentes frações: (i) uma fração ativa, caracterizada pelos produtos da decomposição pelos microorganismos, que possui um tempo de residência curto no solo (1-2 anos); (ii) uma fração lenta, mais resistente à decomposição, graças a uma proteção física ou química, possuindo um tempo de residência de algumas décadas; e (iii) uma fração passiva, que se caracteriza por ser física ou quimicamente mais resistente à decomposição, com um tempo de residência mais longo, da ordem de milênios (Parton et al., 1989). Esse tipo de divisão da matéria orgânica do solo é o mais utilizado na maioria dos modelos desenvolvidos recentemente (Jenkinson \& Rayner, 1977; Veen \& Paul, 1981; Trumbore, 1993).

\section{Submodelo de balanço hídrico, lixiviação e tempera- tura do solo}

Calcula a evaporação e transpiração mensal, a perda de água no solo, o conteúdo de água nas camadas do solo e o fluxo de saturação de água entre as camadas do solo.

\section{Submodelos de produção da planta}

Estimam a concentração mensal de $\mathrm{C}$ e $\mathrm{N}$ no material vivo e morto da parte aérea das plantas, das raízes vivas, da superfície estrutural e metabólica, e o estoque residual no solo. A produção máxima anual da parte aérea e das raízes, sem limitações de nutrientes, é calculada em razão da precipitação anual. Os submodelos de produção primária possuem parâmetros estabelecidos na maioria das principais culturas (milho, soja, trigo, sorgo) e em ecossistemas de pradarias, florestas e savanas ao redor do mundo (Parton et al., 1993).

Além destes submodelos, existem os que representam diferentes práticas de manejo, como: fogo, irrigação, taxas de fertilizantes aplicadas.

\section{Parametrização}

Neste trabalho, simularam-se os fluxos de C considerando-se um sistema solo-planta nas condições de floresta, pastagem e cana-de-açúcar. Os principais dados de entrada utilizados foram: dados climáticos (médias mensais da temperatura máxima e da temperatura mínima do ar e precipitação mensal, de janeiro a dezembro); dados analíticos de plantas (teor de lignina e teor de $\mathrm{N}$ na planta) e dados analíticos de solo $(\mathrm{pH}$, textura, teores de $\mathrm{C}$ e $\mathrm{N}$ no solo). Os dados climáticos e a textura do solo são os que promovem alterações relevantes nas simulações. Nas Tabelas 1, 2 e 3 são apresentados alguns dos parâmetros utilizados, bem como seus respectivos valores e a fonte de origem de cada um deles. Com relação aos dados climáticos, utilizou-se a média mensal de 1953-1992, obtidos do Posto Meteorológico da Escola Superior de Agricultura Luiz de Queiroz/USP.

Das variáveis de saída oferecidas pelo modelo, duas, fundamentais, foram selecionadas para serem analisadas e discutidas nas simulações: (1) concentração de C na matéria orgânica do solo e (2) produção primária líquida. Essas variáveis foram escolhidas por serem importantes nos estudos biogeoquímicos e também porque já existem alguns trabalhos realizados em regiões tropicais ou subtropicais cujos resultados auxiliaram na validação do modelo.

\section{Simulações}

Na bacia do rio Piracicaba, a vegetação original foi substituída principalmente pelo cultivo de cana-de-açúcar e pastagem (Fig. 1). Para verificar os possíveis efeitos das mudanças no uso da terra no estoque de $\mathrm{C}$ e na produção primária líquida, foram realizadas três simulações (Fig. 2)

A primeira considerou a existência de uma floresta por um período longo, estabelecido em 7.000 anos, de forma a se obter através do modelo o seu estado de equilíbrio. Os valores obtidos com relação às diferentes frações de $\mathrm{C}$ no solo foram comparados com dados de literatura, para sua validação, e serviram como variáveis de entrada para as outras simulações que envolveram mudança no uso da terra. Simularam-se, então, condições em que houve o corte da floresta, queima da vegetação remanescente e introdução de uma nova cultura.

Em uma segunda situação, simulou-se a conversão de uma área de floresta para cana-de-açúcar por períodos de 12 e 50 anos, no intuito de avaliar os efeitos da derrubada, queima e cultivo, sobre a matéria orgânica do solo e a produção da cultura. 
Na terceira situação, simulou-se a transição de uma floresta subtropical para uma área de pastagem por um período de 20 anos e, finalmente, a transição para uma área de cultivo de cana-de-açúcar por mais 20 anos.

\section{Simulação floresta $\rightarrow$ cana-de-açúcar}

Nessa simulação, foram utilizados como valores iniciais as concentrações de $\mathrm{C}$ e $\mathrm{N}$ existentes nas diferentes frações da matéria orgânica do solo, obtidas na condição de floresta subtropical em equilíbrio. Em relação à cultura da cana-de-açúcar, considerou-se o seguinte manejo: adubação nitrogenada $\left(100 \mathrm{~kg} \mathrm{ha}^{-1} \mathrm{ano}^{-1} \mathrm{de} \mathrm{N}\right)$, remoção anual da cana-de-açúcar sempre após a queimada da palha e o replantio da cultura a cada cinco anos.

Os parâmetros utilizados na simulação de cana-de-açúcar, em condições subtropicais, são apresentados na

TABELA 1. Valores do modelo CENTURY utilizados nas simulações de florestas subtropicais.

\begin{tabular}{lcl}
\hline Parâmetro & Valor & Fonte \\
\hline Fração de areia no solo & 0,30 & Cerri (1986) \\
Fração de silte no solo & 0,25 & Cerri (1986) \\
Fração de argila no solo & 0,45 & Cerri (1986) \\
Densidade do solo $\left(\mathrm{g} \mathrm{cm}^{-3}\right)$ & 1,3 & Cerri (1986) \\
Número total de camadas no solo & 9 & Pulliam (1996) \\
Número de camadas disponíveis para crescimento da planta & 9 & Pulliam (1996) \\
Fração do excesso de água perdida pela drenagem & 0,75 & Pulliam (1996) \\
pH do solo & 5,7 & Cerri (1986) \\
Valor inicial do C na fração ativa da MO da superfície $\left(\mathrm{g} \mathrm{m}^{-2}\right)$ & 27 & Cerri (1986) \\
Valor inicial do C na fração ativa da MO do solo $\left(\mathrm{g} \mathrm{m}^{-2}\right)$ & 194 & Cerri (1986) \\
Valor inicial do C na fração lenta da MO do solo $\left(\mathrm{g} \mathrm{m}^{-2}\right)$ & 4.145 & Cerri (1986) \\
Valor inicial do C na fração passiva da MO do solo $\left(\mathrm{g} \mathrm{m}^{-2}\right)$ & 2.001 & Cerri (1986) \\
Razão inicial de C/N na fração ativa MO da superfície & 20 & Trumbore et al. (1995) \\
Razão inicial de C/N na fração ativa MO do solo & 10 & Trumbore et al. (1995) \\
Razão inicial de C/N na fração lenta MO do solo & 19 & Trumbore et al. (1995) \\
Razão inicial de C/N na fração passiva MO do solo & 10 & Trumbore et al. (1995) \\
Valor inicial do resíduo da planta na superfície & 119 & Trumbore et al. (1995) \\
Valor inicial do resíduo da planta no solo & 36 & Trumbore et al. (1995) \\
Razão inicial de C/N da liteira da superfície e do solo & 120 & Trumbore et al. (1995) \\
Conc. inicial de lignina nos galhos, caule e raízes grossas mortas & 0,35 & Trumbore et al. (1995) \\
Produção bruta máxima da floresta (g m mês $^{-1}$ de biomassa) & 1.500 & Pulliam (1996) \\
Produção líquida máxima da floresta (g m mês $^{-1}$ me C) & 400 & Pulliam (1996) \\
\hline
\end{tabular}

TABELA 2. Valores do modelo CENTURY utilizados nas simulações da cultura de cana-de-açúcar.

\begin{tabular}{lcl}
\hline Parâmetro & Valor & Fonte \\
\hline Número total de camadas no solo & 5 & Pulliam (1996) \\
Número de camadas disponíveis para crescimento da planta & 4 & Pulliam (1996) \\
Valor inicial do C na fração ativa da MO da superfície $\left(\mathrm{g} \mathrm{m}^{-2}\right)$ & 10,03 & Metherell et al. (1993) \\
Valor inicial do C na fração ativa da MO do solo $\left(\mathrm{g} \mathrm{m}^{-2}\right)$ & 118,78 & Metherell et al. (1993) \\
Valor inicial do C na fração lenta da MO do solo $\left(\mathrm{g} \mathrm{m}^{-2}\right)$ & 3.036 & Metherell et al. (1993) \\
Valor inicial do C na fração passiva da MO do solo $\left(\mathrm{g} \mathrm{m}^{-2}\right)$ & $2.679,3$ & Metherell et al. (1993) \\
Produção bruta máxima da cana-de-açúcar $\left(\mathrm{g} \mathrm{m}^{-2} \mathrm{mês}^{-1}\right.$ de biomassa) & 1.000 & Vallis et al. (1996) \\
Produção líquida máxima da cana-de-açúcar $\left(\mathrm{g} \mathrm{m}^{-2} \mathrm{mês}^{-1} \mathrm{de} \mathrm{C)}\right.$ & 400 & Vallis et al. (1996) \\
\hline
\end{tabular}


TABELA 3. Valores do modelo CENTURY utilizados nas simulações de pastagem.

\begin{tabular}{lcl}
\hline Parâmetro & Valor & Fonte \\
\hline Número total de camadas no solo & 4 & Pulliam (1996) \\
Número de camadas disponíveis para crescimento da planta & 4 & Pulliam (1996) \\
Valor inicial do C na fração ativa da MO da superfície $\left(\mathrm{g} \mathrm{m}^{-2}\right)$ & 10,03 & Metherell et al. (1993) \\
Valor inicial do C na fração ativa da MO do solo $\left(\mathrm{g} \mathrm{m}^{-2}\right)$ & 118,78 & Metherell et al. (1993) \\
Valor inicial do C na fração lenta da MO do solo $\left(\mathrm{g} \mathrm{m}^{-2}\right)$ & 3036 & Metherell et al. (1993) \\
Valor inicial do C na fração passiva da MO do solo $\left(\mathrm{g} \mathrm{m}^{-2}\right)$ & 2679,3 & Metherell et al. (1993) \\
\hline
\end{tabular}

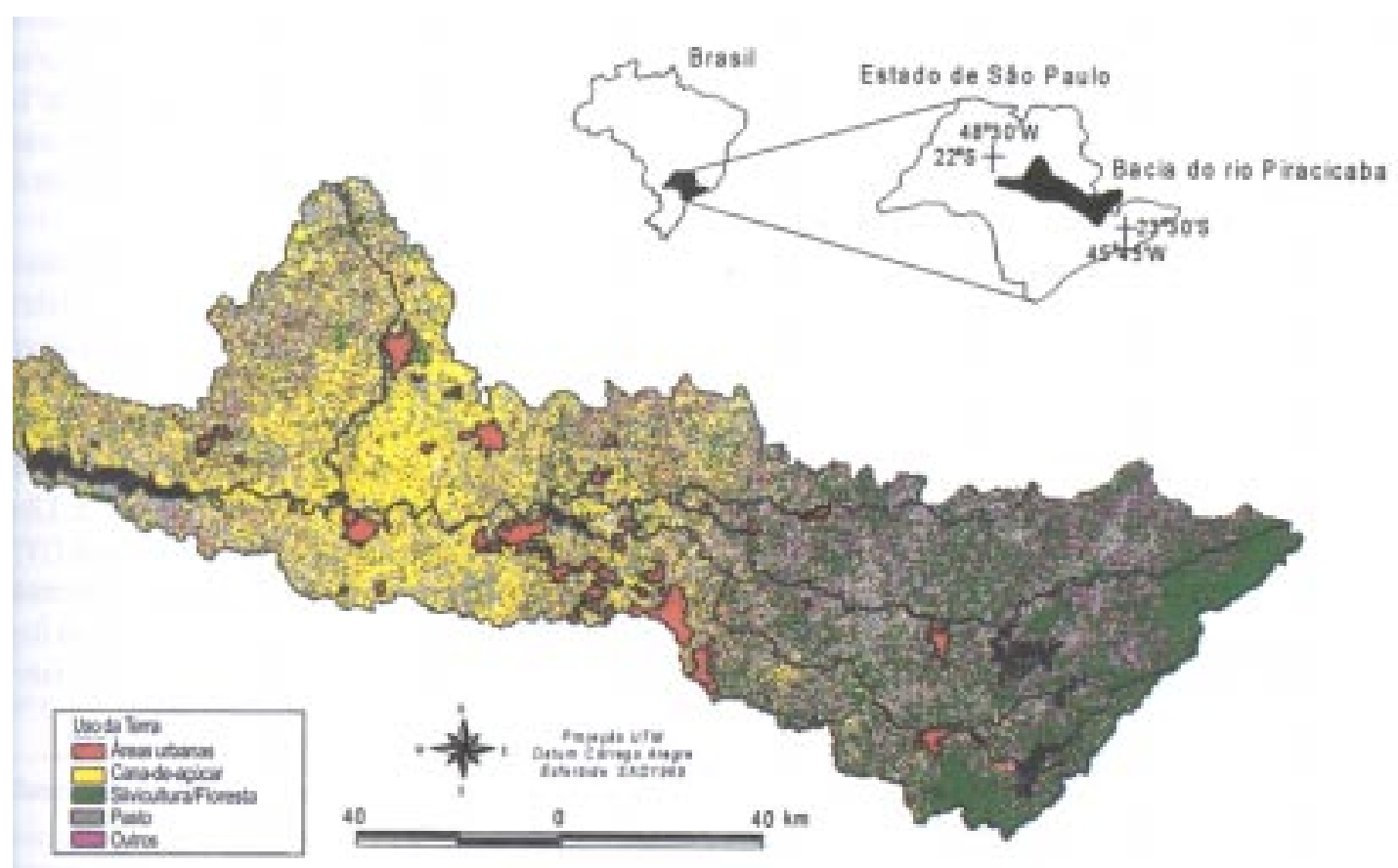

FIG. 1. Mapa de uso da terra na bacia do rio Piracicaba em 1993.

Tabela 2. Quanto aos demais parâmetros do modelo, mantiveram-se os valores utilizados por Vallis et al. (1996), que simularam a introdução da cultura da cana-de-açúcar em regiões da Austrália. Ressalta-se que os parâmetros que não foram alterados são específicos de cana-de-açúcar.

\section{Simulação floresta $\rightarrow$ pasto $\rightarrow$ cana-de-açúcar}

A partir da condição de floresta subtropical em equilíbrio, como citado anteriormente, simulou-se a transformação para pastagem por um período de 20 anos e cultivo da cana-de-açúcar por mais 20 anos, utilizando-se as mesmas condições de cultivo da simulação anterior relati- 
vas à cana-de-açúcar. Os parâmetros que foram alterados para as condições subtropicais, na simulação de pastagem, são apresentados na Tabela 3. Quanto aos demais parâmetros do modelo, mantiveram-se os valores originais do modelo CENTURY 4.0.

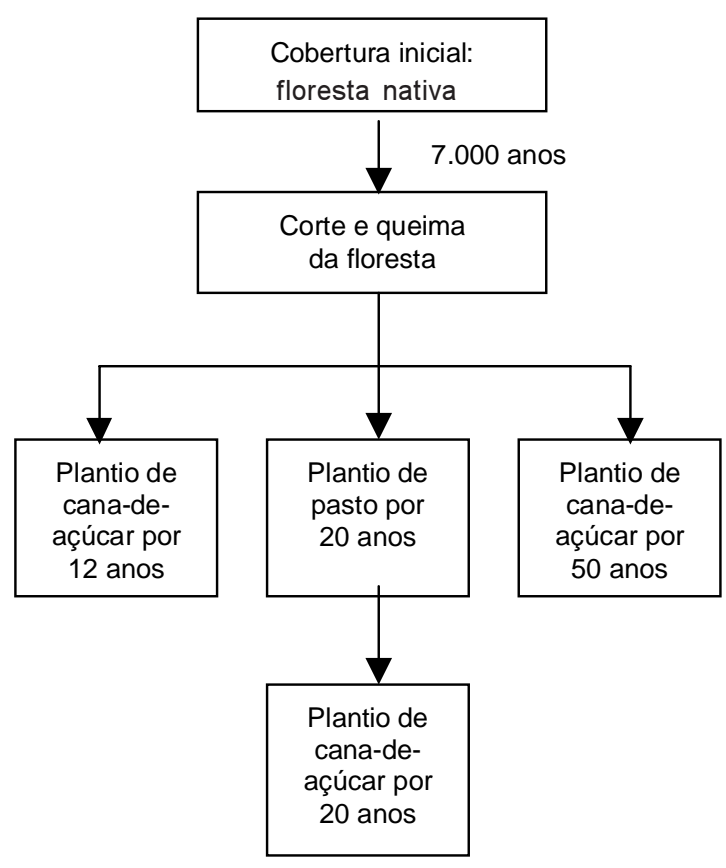

FIG. 2. Esquema das simulações realizadas com o modelo CENTURY.

\section{RESULTADOS E DISCUSSÃO}

Os resultados obtidos nas diferentes simulações podem ser observados na Tabela 4

Em uma floresta subtropical, o valor estimado pelo modelo, referente ao estoque total de $\mathrm{C}$ no solo até $20 \mathrm{~cm}$ de profundidade, foi de $61,5 \mathrm{t} \mathrm{ha}^{-1}$ (Tabela 4). Comparando-se com o valor de $71,9 \mathrm{t} \mathrm{ha}^{-1}$ (Tabela 5) obtido por Cerri (1986) em uma floresta subtropical na bacia do rio Piracicaba, observou-se uma diferença de $14 \%$. Deve-se ressaltar que na simulação realizada com o modelo CENTURY, com relação à floresta subtropical, consideraram-se as mesmas condições de solo da região estudada por Cerri (1986). Quando se comparou com a média de valores observados no que se referem a florestas tropicais $\left(66 \mathrm{t} \mathrm{ha}^{-1} \mathrm{de} \mathrm{C}\right)$, verificou-se que o valor estimado encontrava-se bem próximo da média. Esta diferença pode ser considerada como irrelevante, pois a variabilidade natural do estoque de $\mathrm{C}$ no solo situa-se em torno de 30\% (Tabela 5).

$\mathrm{O}$ valor da produção primária líquida referente à floresta subtropical em condição de equilíbrio foi de $6,6 \mathrm{t} \mathrm{ha}^{-1} \mathrm{ano}^{-1}$. Esse resultado é próximo ao valor de 7,6 tha $^{-1}$ ano $^{-1}$, encontrado por Weaver \& Murphy (1990) em uma floresta tropical de Porto Rico. Comparando-se com algumas estimativas feitas em florestas tropicais obtidas por Whittaker \& Likens (1975) de 9,0 tha-1 $\mathrm{ano}^{-1}$, ou por Atjay et al. (1979), de $10,0 \mathrm{tha}^{-1} \mathrm{ano}^{-1}$, o valor simulado por esse modelo foi cerca de $30 \%$ menor. A produção menor na floresta subtropical pode ser justificada pelo fato de

TABELA 4. Comparação das frações de matéria orgânica do solo e produção primária em simulações realizadas para diferentes usos da terra.

\begin{tabular}{|c|c|c|c|c|c|c|}
\hline \multirow[t]{2}{*}{ Tipo de uso da terra } & \multicolumn{3}{|c|}{$\begin{array}{l}\text { Frações da matéria orgânica } \\
\text { do solo }\end{array}$} & \multirow[t]{2}{*}{ Total } & \multirow{2}{*}{$\begin{array}{l}\text { Produção } \\
\text { primária } \\
\text { líquida }\end{array}$} & \multirow{2}{*}{$\begin{array}{c}\text { Produtividade } \\
\text { primária } \\
\text { líquida }\end{array}$} \\
\hline & Ativa & Lenta & Passiva & & & \\
\hline & \multicolumn{6}{|c|}{ (t ha $\left.{ }^{-1} \mathrm{ano}^{-1} \mathrm{de} \mathrm{C}\right)$} \\
\hline Floresta & 1,24 & 31,81 & 26,79 & 61,5 & - & 6,6 \\
\hline Cana-de-açúcar (12 anos) & 0,64 & 16,16 & 26,56 & 44,0 & 77 & - \\
\hline Cana-de-açúcar (50 anos) & 0,44 & 9,33 & 25,08 & 35,4 & 82 & - \\
\hline Pasto (20 anos) & 1,38 & 20,32 & 23,97 & 47,0 & - & 6,5 \\
\hline Cana-de-açúcar (20 anos) & 0,54 & 11,79 & 23,47 & 36,5 & 80 & - \\
\hline
\end{tabular}


regiões tropicais apresentarem temperaturas e precipitações maiores e mais constantes que regiões subtropicais.

Quando da substituição da floresta por canade-açúcar, verificou-se uma diminuição na concentração de carbono nas diferentes frações da matéria orgânica do solo após alguns anos de instalação da cultura (Tabela 4).

$\mathrm{O}$ estoque total de $\mathrm{C}$ no solo cultivado com cana-de-açúcar, simulado pelo modelo, após um período de 12 anos, foi de 44,0 t ha ${ }^{-1}$, valor muito próximo ao obtido por Cerri (1986) de 44,6 t ha-1. No tocante ao cultivo de cana-de-açúcar por um período de 50 anos, o valor simulado foi de 35,4 t ha-1, $8 \%$ menor em relação ao valor obtido por Cerri (1986) de 38,5 t ha-1. Davidson \& Ackerman (1993), baseando-se em uma extensa revisão da literatura de experimentos de campo, estimaram que o estoque de $\mathrm{C}$ no solo quando da alteração de floresta para instalação de culturas decresceu em cerca de $30 \%$. Os resultados obtidos por Vitorello et al. (1989) seguiram a mesma tendência. As simulações realizadas neste trabalho também chegaram a resultados semelhantes, ou seja, um decréscimo de $28 \%$ no estoque de $\mathrm{C}$ nos primeiros 12 anos e diminuição de $42 \%$ em 50 anos de cultivo do solo com cana-de-açúcar.
Para analisar o componente funcional da floresta, foi considerada a produção primária líquida (Tabela 4), enquanto no que tange à cana-de-açúcar considerou-se a variável que mais interessa ao produtor, isto é, a produção de colmos. A produção de cana-de-açúcar apresentou média de $82 \mathrm{t} \mathrm{ha}^{-1} \mathrm{ano}^{-1}$, considerando o período de 50 anos de cultivo, valor similar à produção média de cana-de-açúcar no Estado de São Paulo, que varia de 70 a $100 \mathrm{t} \mathrm{ha}^{-1}$ ano $^{-1}$ (Carvalho, 1996). Apesar de os valores simulados de produção mostrarem-se similares aos valores observados no campo, o padrão de distribuição da produção da cultura de cana-de-açúcar ao longo do ciclo de replantio a cada cinco anos não apresentou resultados condizentes com a realidade. Na maioria dos casos, esperar-se-ia que a produção de cana-de-açúcar tivesse um valor maior de produção no início de cada ciclo, e que fosse diminuindo ao longo desse período (Fig. 3a). Observou-se, no entanto, que a principal variável que influencia a produção é a precipitação anual, ou seja, produção elevada nos anos de maiores chuvas. $\mathrm{O}$ mesmo tipo de comportamento na produção de cana-de-açúcar foi observado por Keating et al. (1994) em simulações realizadas com o modelo CENTURY em algumas regiões da Austrália. Esse padrão resulta do modo como o CENTURY trata a cana-de-açúcar, não considerando a rebrota da

TABELA 5. Valores quantificados de estoque de carbono no solo em florestas tropicais e subtropicais.

\begin{tabular}{lcl}
\hline Local & $\begin{array}{c}\text { Estoque de carbono no solo } \\
\left(\mathrm{t} \mathrm{ha}^{-1}\right)\end{array}$ & Referência \\
\hline Piracicaba & 61,5 & Este estudo (CENTURY) \\
Piracicaba & 71,9 & Cerri (1986) \\
Manaus & 71,0 & Trumbore (1993) \\
Rondônia & 43,5 & Moraes (1995) \\
Manaus & 66,0 & Choné et al. (1991) \\
Manaus & 90,0 & Choné et al. (1991) \\
Porto Rico (CENTURY) & 88,0 & Pulliam (1996) \\
Porto Rico & 53,0 & Pulliam (1996) \\
Costa Rica & 75,0 & Veldkamp (1994) \\
Costa Rica & 41,5 & Veldkamp (1994) \\
\hline Média & 66,0 & \\
\hline
\end{tabular}


cultura. Seriam necessários ajustes na rotina de produção do modelo.

A simulação da transição de uma floresta para pasto, seguida do cultivo da cana-de-açúcar, mostrou um decréscimo na concentração de $\mathrm{C}$ nas diferentes frações da matéria orgânica do solo similar ao encontrado na simulação de floresta com relação a cana-de-açúcar. Na primeira mudança (florestapastagem), a perda de $\mathrm{C}$ foi de $24 \%$, enquanto na segunda alteração (pastagem-cana) a perda foi de $22 \%$.

Existem dois fatores que contribuem para a diminuição da concentração de $\mathrm{C}$ no solo: erosão $\mathrm{e}$ mineralização da matéria orgânica do solo. Estima-se que cerca de $80 \%$ da área cultivada do Estado de São Paulo esteja sofrendo processo erosivo além dos limites de tolerância (Cerri, 1997). Portanto, esse processo pode contribuir para a perda de $\mathrm{C}$ durante $\mathrm{o}$ cultivo. Por outro lado, a mineralização da matéria orgânica do solo seria acelerada com o cultivo, em face do processo de preparo do solo, que propicia maior contato da matéria orgânica com o ar (oxigênio).

Nas várias simulações efetuadas foram sempre observadas maiores perdas de $\mathrm{C}$ nos primeiros anos de cultivo (Tabela 4). Essa diminuição mais intensa no início do cultivo também foi observada por Cerri (1986), Mann (1986) e Davidson \& Ackerman (1993).

Quanto à produção primária do pasto, o valor médio simulado foi de $6,5 \mathrm{tha}^{-1}$, enquanto o valor médio da produção de cana-de-açúcar foi novamente de $80 \mathrm{t} \mathrm{ha}^{-1}$ ano $^{-1}$, dentro da faixa de variação da produção média no Estado de São Paulo (Tabela $4 \mathrm{e}$ Fig. 3b e 3c).

Os resultados das simulações indicaram que a quantidade e a qualidade da matéria orgânica do solo é modificada pelo cultivo. Essas modificações podem conduzir a mudanças nas propriedades químicas, físicas e biológicas do solo. Assim sendo, através de práticas de manejo adequadas, deve-se evitar ao máximo as perdas de $\mathrm{C}$ do solo, principalmente nos primeiros anos de cultivo, quando as ocorrências são maiores.
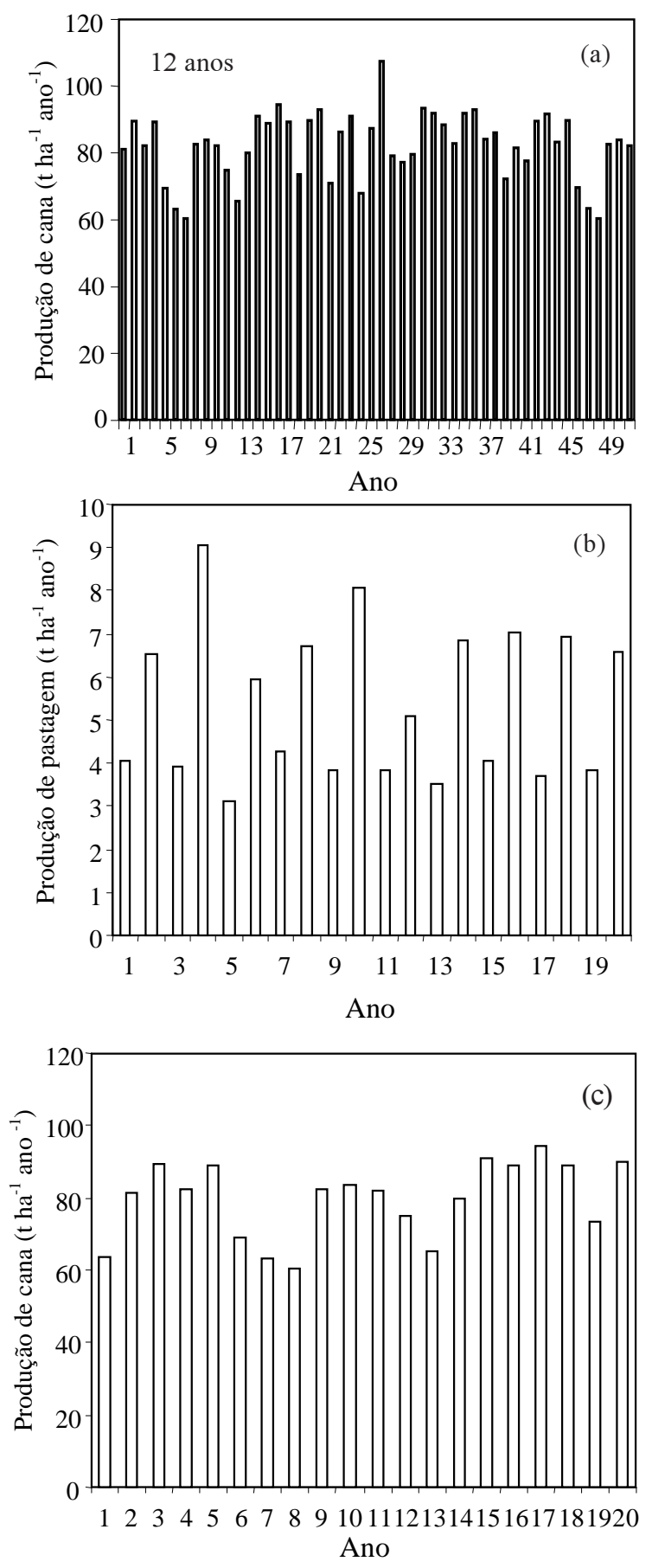

FIG. 3. Resultados da simulação para a produção primária líquida para (a) cana-de-açúcar 12 e 50 anos, (b) pastagem por 20 anos, (c) canade-açúcar para 20 anos. 


\section{CONCLUSÕES}

1. As simulações realizadas nos cenários de floresta subtropical, pastagem e cana-de-açúcar apresentam resultados de produção primária e de estoque total de $\mathrm{C}$ no solo próximos aos observados no campo por diferentes autores.

2. Os valores simulados de produção relativos à cana-de-açúcar são similares aos valores observados no campo, porém o padrão de distribuição da produção da cultura de cana-de-áçucar ao longo do ciclo de replantio a cada cinco anos não apresenta resultados condizentes com a realidade.

3. Os resultados das simulações indicam que a quantidade da matéria orgânica do solo é modificada pelo tipo de cultivo. Com práticas de manejo adequadas, pode-se evitar ao máximo as perdas de $\mathrm{C}$ do solo, principalmente nos primeiros anos de cultivo.

\section{REFERÊNCIAS}

ATJAY, G.L.; KETNER, P.; DUVIGNEAUD, P Terrestrial primary production and phytomass. In: BOLIN, B.; DEGENS, E.T.; KEMPE, S.; KETNER, P. (Eds.). The global carbon cycle. Chichester : J Wiley, 1979. p.129-181.

BONAN, G.B. Land-atmosphere $\mathrm{CO}_{2}$ exchange simulated by a land surface process model coupled to an atmospheric general circulation model. Journal of Geophysical Research, Washington, n.100, p.2817-2831, 1995

CARTER, M.R.; PARTON, W.J.; ROWLAND, I.C.; SCHULTZ, J.E.; STEED, G.R. Simulation of soil organic carbon and nitrogen changes in cereal and pasture systems of southern Australia. Australian Journal of Soil Research, Melbourne, v.31, p.481-491, 1993.

CARVALHO, L.C. A visão do setor sucroalcooleiro. In FERNADES, E.S.L.; COELHO, S.T. (Eds.). Perspectivas do álcool combustível no Brasil. São Paulo : USP-Instituto de Eletrônica e Energia, 1996. $166 \mathrm{p}$

CERRI, C.C. Dinâmica da matéria orgânica do solo no agrossistema cana-de-açúcar. Piracicaba USP-ESALQ, 1986. 197p. Tese de Livre-Docência

CERRI, C.E.P. Perdas de solo por erosão em áreas agrícolas da bacia do rio Piracicaba, através do geoprocessamento: análise exploratória. Piracicaba USP-ESALQ, 1997. 55p.

CHONÉ, T.; ANDREX, F.; CORREA, J.C.; VOLKOFF, B.; CERRI, C.C. Changes in organic matter in an oxisol from the central Amazonia forest during eight years as pasture, determined by ${ }^{13} \mathrm{C}$ isotopic composition. In: BERTHELIN, J. (Ed.). Diversity of environmental biogeochemistry. Amsterdam : Elsevier, 1991. p.307-405.

DAVIDSON, E.A.; ACKERMAN, I.L. Changes in soil carbon inventories following cultivation of previously untilled soils. Biogeochemistry, Dordrecht, v.20, p.161-193, 1993.

JENKINSON, D.S.; RAYNER, J.H. The turnover of soil organic matter in some of the Rothamsted classical experiments. Soil Science, Baltimore, v.123, p.298-305, 1977.

KEATING, B.A.; VALLIS, I.; PARTON, W.J.; CATCHPOOLE, V.R.; MUCHOW, R.C.; ROBERTSON, M.J.; EGAN, B.T. Modelling and its application to nitrogen management and research for sugarcane. In: CONFERENCE OF THE AUSTRALIAN SOCIETY OF SUGAR CANE TECHNOLOGIS T S, 1994 , Townsville Proceedings. Brisbane : W. Ferguson, 1994. p.131-142.

McNULTY, S.G.; VOSE, J.M.; SWANK, W.T. Scaling predicted pine forested hydrology and productivity across the Southern United States. In: QUATTROCHI, D.A.; GOODCHILD, M.F. (Eds.). Scale in remote sensing and GIS. New York Lewis, 1997. p.187-209.

MANN, L.K. Changes in soil carbon storage after cultivation. Soil Science, Baltimore, v.142, p.279-288, 1986.

MELILLO, J.M.; McGUIRE, A.D.; KICKLIGHTER, D.W.; MOORE III, B.; VOROSMARTY, C.J.; SCHLOSS, A.L. Global climate change and terrestrial net primary production. Nature, London, v.363, n.6426, p.234-240, 1993.

METHERELL, A.K.; HARDING, L.A.; COLE, C.E.; PARTON, W.J. CENTURY soil organic matter model : agroecosystem version 4.0. Fort Collins : Colorado State University, 1993. 1v. (GPSR Technical Report, 4).

MORAES, J.F.L. Propriedades do solo e dinâmica da matéria orgânica associadas às mudanças do uso 
da terra em Rondônia (RO)/Brasil. Piracicaba USP-CENA, 1995. 69p. Tese de Doutorado.

PARTON, W.J.; RASMUSSEN, P.E. Long-term effects of crop management in wheat-fallow: II. Century model simulations. Soil Science Society of America. Journal, Madison, v.58, p.530-536, 1994.

PARTON, W.J.; SANFORD, R.L.; SANCHEZ, P.A.; STEWART, J.W.B.; BONDE, T.A.; CROSLEY, D; VEEN, H. van; YOST, R. Modeling soil organic matter dynamics in tropical soils. In: COLEMAN, D.C.; OADES, J.M.; UEHARA, G. (Eds.). Dynamics of soil organic matter in tropical ecosystems. Honolulu : University of Hawaii Press, 1989. p.153-171.

PARTON, W.J.; SCHIMEL, D.S.; COLE, C.V.; OJIMA, D.S. Analysis of factors controlling soil organic levels of grasslands in the Great Plains. Soil Science Society of America. Journal, Madison, v.51, p.1173-1179, 1987

PARTON, W.J.; SCURLOCK, J.M.O; OJIMA, D.S.; GILMANOV, T.G.; SCHOLES, R.J.; SCHIMEL, D.S.; KIRCHNER, T.; MENAULT, J.C.; SEASTEDT, T.; GARCIA MOYA, E.; KAMNALRUT, A.; KINYAMARIO, J.I Observations and modeling of biomass and soil matter dynamics for the grassland biome worldwide. Global Biogeochemical Cycles, Washington, v.7, p.785-809, 1993.

PARTON, W.J.; STEWART, J.W.B.; COLE, C.V Dynamics of C, N, P and S in grassland soil: a model. Biogeochemistry, Dordrecht, v.8, p.109-131, 1988

PELLEGRINO, G.Q. Análise espaço-temporal de dados hidrológicos da bacia do rio Piracicaba. Piracicaba : USP-ESALQ, 1995. 117p. Tese de Mestrado.

POTTER, C.S.; RANDERSON, J.T.; FIELD, C.B.; MATSON, P.A.; VITOUSEK, P.M.; MOONEY, H.A.; KLOOSTER, S.A. Terrestrial ecosystem production: a process model based on global satellite and surface data. Global Biogeochemical Cycles, Washington, v.7, n.4, p.811-841, 1993.

PULLIAM, W.M. Forest century parameterization workbook. Fort Collins : Colorado State University, 1996. 29p.

SANFORD JUNIOR, R.L.; PARTON, W.J.; OJIMA, D.S.; LODGE, D.J. Hurricane effects on soil organic matter dynamics and forest production in the Luquillo
Experimental Forest, Puerto Rico: results of simulation modelling. Biotropica, Saint Louis, v.23, n.4a, p.364-372, 1991.

SÃO PAULO (Estado). Conselho Estadual de Recursos Hídricos. Plano integrado de recursos hídricos: primeiro plano do Estado de São Paulo. São Paulo : DAEE, 1990. 140p

SÃO PAULO (Estado). Secretaria do Meio Ambiente Bacia do rio Piracicaba: estabelecimento de metas ambientais e reenquadramento dos corpos d'água. São Paulo, 1994. 81p. (Série Relatórios).

SCHIMEL, D.S.; BRASWELL, B.H.; McKEOWN, R.; OJIMA, D.S.; PARTON, W.J.; PULLIAM, W Climate and nitrogen controls on the geography and timescales of terrestrial biogeochemical cycling. Global Biogeochemical Cycles, Washington, v.10, n.4, p.677-692, 1996

STEYAERT, L.T. Environmental simulation modeling In: GOODCHILD, M.F.; PARKS, B.O.; STEYAERT, L.T. (Eds.). Environmental modeling with GIS. Oxford : Oxford University Press, 1993. p. 105-107

TRUMBORE, S. Comparison of carbon dynamics in tropical and temperate soils using radiocarbon measurements. Global Biogeochemical Cycles, Washington, v.7, p.275-290, 1993.

TRUMBORE, S.E.; DAVIDSON, E.A.; CAMARGO, P.B.; NEPSTAD, D.C.; MARTINELLI, L.A. Belowground cycling of carbon in forests and pastures of eastern Amazonia. Global Biogeochemical Cycles, Washington, v.9, p.515-528, 1995.

VALLIS, I;; PARTON, W.J.; KEATING, B.A.; WOOD, A.W. Simulation of the effects of trash and N fertilizer management on soil organic matter levels and yields of sugarcane. Soil and Tillage Research, Amsterdam, v.38, p.115-132, 1996.

VEEN, J.A. van; PAUL, E.A. Organic carbon dynamics in grassland soils. 1. Background information and computer simulation. Canadian Journal of Soil Science, Otawa, v.61, p.185-201, 1981

VELDKAMP, E. Organic carbon turnover in three tropical soils under pasture after deforestation. Soil Science Society of America. Journal, Madison, v.58, p.175-180, 1994.

VITORELLO, V.A.; CERRI, C.C.; ANDREUX, F.F.C.; VICTORIA, R.L. Organic matter and natural carbon-13 distribution in forested and cultivated 
oxisols. Soil Science Society of America. Journal, Madison, v.53, p.773-778, 1989

WARNANT, P.; FRANÇOIS, L.; STRIVAY, D.; GÉRARD, J.C. CARAIB: A global model of terrestrial biological productivity. Global Biogeochemical Cycles, Washington, v.8, p.255-270, 1994.

WEAVER, P.L.; MURPHY, P.G. Forest structure and productivity in Puerto Rico's Luquillo Mountains Biotropica, Saint Louis, v.22, p.69-82, 1990
WHEELER, D.J. Commentary: linking environmental models with geographic information system for global change research. Photogrammetric Engineering and Remote Sensing, Falls Church, v.59, n.10, p.1497-1501, 1993.

WHITTAKER, R.H.; LIKENS, G.E. Primary production: the biosphere and man. In: LEITH, H.; WHITTAKER, R.H. (Eds.). Primary productivity of the biosphere. Berlin : Springer-Verlag, 1975 p.305-328 